\title{
Sulforaphane Protects against Brain Diseases: Roles of Cytoprotective Enzymes
}

Sun $Y^{1}$, Yang $T^{1}$, Mao $L^{2}$ and Zhang $F^{1,2 *}$

${ }^{1}$ Department of Neurology, University of Pittsburgh

School of Medicine, USA

${ }^{2}$ Key Lab of Cerebral Microcirculation, Taishan Medical University, China

*Corresponding author: Feng Zhang, Department of Neurology, University of Pittsburgh, Pittsburgh, PA, 15213, USA

Received: December 25, 2016; Accepted: February 14, 2017; Published: February 16, 2017

\begin{abstract}
Sulforaphane (SFN) is a kind of isothiocyanate derived from broccoli and other cruciferous vegetables. Because of its roles of antioxidant, antiinflammatory, and anti-tumor through multiple targets and various mechanisms, SFN has drawn broad attention of the researchers. One of the most important target of SFN is nuclear factor erythroid 2 related factor 2 (Nrf2), wildly known for its ability to regulate the expression of a series of cytoprotective enzymes with antioxidative, prosurvival, and detoxification effects. Multiple researches have shown that SFN protects against central nervous system diseases through Nrf2pathway. In this article, we list SFN contents in common cruciferous vegetables, and summarize recent advances in the protective effects of SFN against acute brain injuries and neurodegenerative diseases through activating Nrf2 signaling pathway.
\end{abstract}

Keywords: Sulforaphane; Nrf2; Stroke; TBI; Dementia; Autism

\section{Abbreviations}

AD: Alzheimer's Disease; ARE: Antioxidant Response Elements; ASD: Autism Spectrum Disorders; BBB: Blood-Brain Barrier; CNS: Central Nervous System; EGCG: Epigallocatechin Gallate; GSH: Glutathione; GSK-3 $\beta$ : Glycogen Synthase Kinase-3 $\beta$; GST: Glutathione S-Transferase; HO-1: Heme Oxygenase 1; ICH: Intracerebral Hemorrhages; KEAP1: Kelch-Like Ech Associated Protein 1; NQO-1: NADPH Quinine Oxidoreductase 1; Nrf2: Nuclear Factor Erythroid 2 Related Factor; OGD: Oxygen-Glucose Deprivation; PD: Parkinson's Disease; SFN: Sulforaphane; TBI: Traumatic Brain Injury

\section{Introduction}

Central nervous system (CNS) diseases are major health problems because of their high incidence of death and disability around the world. Multiple pathological processes are involved in brains diseases, including oxidative stress, protein misfolding and aggregation, perturbed calcium homeostasis, excitotoxicity, inflammation, and apoptosis $[1,2]$. In the past decades, there has been a growing interest in a number of dietary phytochemicals for their antioxidant antiinflammatory effects. Among them 1-isothiocyanate-4-methylsulfinyl butane (sulforaphane, SFN) is one of the most popular one [3-5].

SFN is the hydrolysis product of glucoraphanin, the main glucosinolates in broccoli [6,7]. Glucosinolates are wildly present in cruciferous vegetables (Table 1), such as broccoli, brussel sprouts, kale, cabbage, and cauliflower $[8,9]$, but SFN contents are relatively low (Table 2 and Table 3) [10,11]. Upon consumption, however, glucoraphanin is hydrolyzed by myrosinase, and then converted into SFN [12] has been demonstrated that SFN has neuroprotective effects against brain diseases via a number of mechanisms [3]. In general, SFN inhibits phase I enzymes through directly interacting with cytochrome P450 and, on the other hand, induces phase II enzymes through activating nuclear factor E2-factor related factor
(Nrf2). Phase I enzymes are usually involved in oxidation, reduction, or hydrolysis and generally lead to detoxification, while phase II enzymes demonstrate protection effects against CNS diseases due to their antioxidative and detoxifying roles. Here, this review will focus on the protective effects of SFN against various CNS diseases through activating Nrf2.

\section{Bioactivity of SFN}

The molecular weight of SFN is 177. Its chemical structure is shown in Figure 1, where its bioactive group is indicated by an arrow. This isothiocyanate group makes SFN an electrophile and readily reacts with nucleophiles, especially cysteine residues in proteins [13-15]. It is lipophilic and can be passively absorbed by cells, where SFN is rapidly conjugated with glutathione (GSH) by glutathione S-transferases (GSTs), leading to maintenance of a concentration gradient and ensuring the passive diffusion into the cell [5]. Then, it is metabolized sequentially by $\gamma$-glutamyl-transpeptidase, cysteinylglycinease, and $\mathrm{N}$-acetyltransferase, and the derived conjugates are transported into the systemic circulation [14].

Pharmacokinetic studies in both humans and animals show that SFN can achieve micromole concentrations and accumulate in tissues. This process is rapid, with peak plasma concentration between 1 to 3 Table 1: Glucosinolate contents in cruciferous vegetables.

\begin{tabular}{|c|c|}
\hline Raw food $\mathbf{( 1 0 0} \mathbf{g})$ & Glucosinolates $\mathbf{( m g )}$ \\
\hline Broccoli & 61.1 \\
\hline Brussels sprouts & 247 \\
\hline Cabbage & 108.9 \\
\hline Cabbage, red & 66.9 \\
\hline Cauliflower & 62 \\
\hline Kale, curly & 89.4 \\
\hline Kohlrabi & 109.3 \\
\hline Turnip-Swede & 56 \\
\hline
\end{tabular}

Austin J Cerebrovasc Dis \& Stroke - Volume 4 Issue 1 - 2017 ISSN : 2381-9103 | www.austinpublishinggroup.com

Zhang et al. (C) All rights are reserved
Citation: Sun Y, Yang T, Mao L and Zhang F. Sulforaphane Protects against Brain Diseases: Roles of Cytoprotective Enzymes. Austin J Cerebrovasc Dis \& Stroke. 2017; 4(1): 1054. 
Table 2: SFN contents in vegetable juices.

\begin{tabular}{|c|c|}
\hline Juice from & SFN contents $(\mu \mathrm{g} / \mathrm{ml})$ \\
\hline White cabbage & 2.77 \\
\hline Broccoli & 7.77 \\
\hline Red cabbage & 8.94 \\
\hline Cauliflower & 0.66 \\
\hline Brussels sprouts & 4.74 \\
\hline
\end{tabular}

Table 3: SFN contents in vegetable powers (processed at $90^{\circ} \mathrm{C}$ ).

\begin{tabular}{|c|c|}
\hline Vegetable & SFN concentration $(\boldsymbol{\mu g} / \mathbf{g})$ \\
\hline Broccoli & 476.5 \\
\hline Cabbage & 168.4 \\
\hline Chinese cabbage & 129.1 \\
\hline Red cabbage & 91.3 \\
\hline Carrot & 85.3 \\
\hline Choy & 39.2 \\
\hline Cauliflower & 34.6 \\
\hline Radish & 28.9 \\
\hline Chinese white turnip & 17.7 \\
\hline
\end{tabular}

hours after administration of SFN [14]. After a single oral dose of $50 \mu \mathrm{mol}$ of SFN, the peak plasma concentration in rat was about 20 $\mu \mathrm{M}$ [16]; while a dose of $200 \mu \mathrm{mol}$ leads to a peak concentration of 1 $\mu \mathrm{M}$ in human plasma or serum [17]. The elimination process of SFN in both plasma and tissue is also rapidly, within 12-24 hours after administration, majorly through urinary excretion.

Blood-brain barrier (BBB) is a highly selective semi-permeable membranes barrier that separates the circulating blood from the brain extracelluar fluid in the CNS. The ability to go through the BBB is essential to exert neuroprotective effects for any phytochemicals. Several researches have demonstrated that SFN can traverse the BBB and accumulate in the CNS. After SFN gavage to mice, Jazwa, et al. found that SFN was able to cross the BBB and accumulate in cerebral tissues such as the ventral midbrain and striatum [18]. It reached the peak in 15 minutes and disappeared after 2hours. Clarke, et al. also detected SFN in the CNS [19].

\section{Nrf2 signaling pathway}

Nrf2 is a transcription factor with a characteristic Cap ' $n$ ' collar structure $[15,20]$. It is the key regulator of cytoprotective phase II enzymes, including glutathione S-transferase (GST), heme oxygenase 1 (HO-1) and NADPH quinine oxidoreductase 1 (NQO-1), all of which play important roles in anti-oxidation and detoxification [2022]. Kelch-like ECH associated protein 1 (Keap1) and antioxidant response elements (ARE) are another two key factors in Nrf2 signaling pathway.

Under unstressed condition, Nrf2 forms a complex with Keap1, facilitating the ubiquitination and degradation of Nrf2 by the ubiquitin-proteasome system [20,23]. Keap1 is a cysteine-rich protein, and three cysteine residues (Cys151, Cys273, and Cys288) are essential for Keap1-mediated inhibition of Nrf2 activity [20,24]. Under oxidative or electrophilic conditions, however, the cysteine residues of Keap1 will be oxidized, resulting in conformational

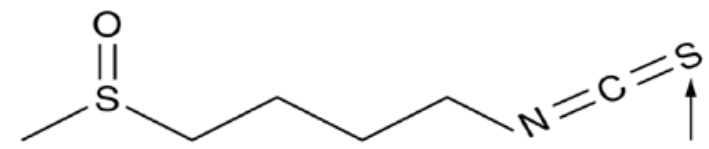

Figure 1: Chemical structure of SFN. The arrow indicates its bioactive group.

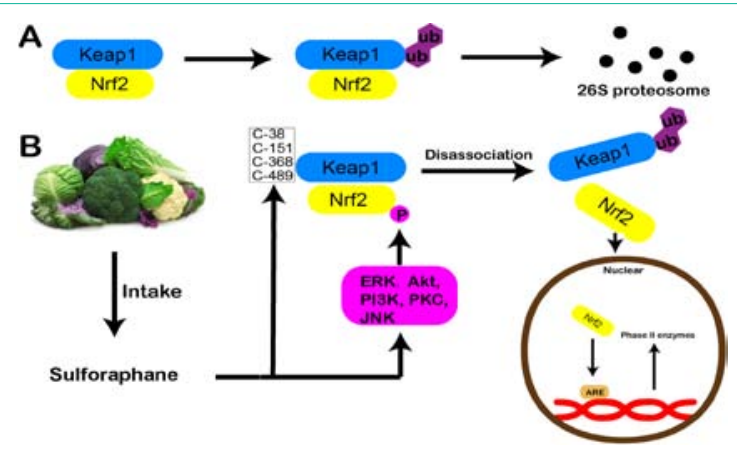

Figure 2: Diagram showing the mechanism that SFN activates Nrf2. (A) Under normal condition, Nrf2 is inactive due to its sequestration by Keap1 and degradation by the proteasome. (B) After eating cruciferous vegetables, SFN is synthesized and absorbed. It then reacts with several cysteine residues in Keap1, including Cys-38, Cys-151, Cys-368, and Cys-489. These reaction results in conformational changes of Keap1, subsequently releasing Nrf2. Nrf2 then translocate into nucleus, binds to the antioxidant response element (ARE) in the promoters phase II enzyme genes.

change of Keap1. This further leads to dissociation of the Nrf2-Keap1 complex, thereby stabilization and nuclear translocation of Nrf2 (Figure 2) [20,23]. Once in the nucleus, Nrf2 forms a heterodimer with a group of nuclear proteins called small Maf proteins [24]. This dimerization increases the binding specificity of Nrf2 to the ARE located in the promoter region of phase II genes [25-27].

In addition to Keap1, recent studies show that there exists another model for Nrf2 ubiquitination and degradation, which is Keap1-independent [28]. In this model, glycogen synthase kinase$3 \beta$ (GSK3 $\beta$ ) phosphorylates two serine residues of Nrf2, Ser342 and Ser347. Phosphorylated Nrf2 can bind with an ubiquitin ligase adaptor $\beta$-TrCP. $\beta$-TrCP links Nrf2 to the Cullin1/Rbx1 ubiquitination complex, which is subsequently degraded. This GSK-3 $\beta$ and $\beta$-TrCPdependent Nrf2 degradation model is supported by the findings that GSK-3 $\beta$ inhibitors stabilize Nrf2 in Keap $1^{-/}$mouse cells [29].

\section{SFN mediate activation of Nrf2}

Direct effects of SFN on Nrf2: SFN can enhance Nrf2 activity via several approaches. It has been reported that SFN increases Nrf2 transcription, probably by reducing methylation of the first $15 \mathrm{CpGs}$ of Nrf2 promoters [30]. Consequently, SFN is reported to upregulates Nrf2 expression cardiac cell [31] and epidermal cells [30]. As an Electrophile, SFN reacts with thiol groups of Keap1 to form thionoacyl adduts, especially C-151 [31,32], which is essential for the association of Cul3 ubiquitin ligase [33]. In addition to C-151, several other sensor cysteines in human Keap1 that can be modified by SFN, including C-38, C-368 and C-489 [34,35]. By modifying these cysteines, SFN can affect the Nrf2/Keap1 complex and prevent ubiquitination of Nrf2. A recent study showed that SFN suppressed the activity of GSK-3 $\beta$ and enhanced Nrf2 nuclear translocation [36]. 
Collectively, SFN stabilizes Nrf2 through antagonizing proteasomedependent degradation [37].

Long-lasting effects of SFN-mediated upregulation of cytoprotective enzymes: Although absorbed SFN is cleared from the body within a few hours, the SFN-mediated effects exist for a long time. Bergström, et al. studied the kinetics of the SFN-induced Nrf2 response in astrocytes [38]. After a 4-hour SFN exposure, there were increases in NQO1 and HO-1 mRNAs that persisted for 24 hours, and the levels of corresponding proteins remained elevated for over 48 hours. Consequently, peroxide-clearing activity and glutathione levels were elevated, resulting in an increased resistance of astrocytes to superoxide-induced damage [38]. In addition, SFN can be taken for a longer period, as it is a dietary component without toxicity. For instance, Bai, et al. [31] showed that diabetic mice treated with SFN for three months showed a reduced incidence of DCM at the end of the treatment and after 6 months [31]. More evidence has been summarized by Angeloni and colleagues [39], which included long-term decreases of intracellular ROS production, increased cell viability, and decreased DNA fragmentation, accompanied by the induction of antioxidants and phase II enzymes. These features of SFN are critical for long-term neuroprotection.

\section{SFN up regulates Nrf2 target proteins}

After Nrf2 pathway activation, over two hundred genes are induced expression and exert detoxification and antioxidant defense. Here our discussion will focus on the phase II enzymes that participate in neuroprotection against common CNS disease.

Antioxidative enzymes: Glutamate cysteine ligase(GCL) is the rate-limiting enzyme in GSH synthesis [40]. In most eukaryotic cells, GCL enzymes are heterodimeric complexes consisting of two distinct gene products, the catalytic subunit (GCLC) and the modifier subunit (GCLM) [41]. SFN induces the expression of both GCLC and GCLM in brain cells, thus enhancing the production of GSH [42]. In addition, Vauzour, et al. [43] observed that SFN could protect against 5-S-cysteinyl-dopamine-induced neurons death, by increasing the expression of GST and glutathione reductase. Similar to these results, Morroni and fellows also suggested SFN could activate Nrf2 signaling pathway, increasing the levels of GHS, GST, and glutathione reductase.

\section{Detoxifying enzymes:}

Heme oxygenase 1 (HO-1): HO-1catalyzes the first and ratelimiting step of heme degradation, and HO-1 protects cells through a net reduction in superoxide and other reactive oxygen species [44]. Many researchers have demonstrated that SFN could induce HO-1 expression via activating Nrf2 signaling pathway. Alfier and colleagues demonstrated that SFN treatment activated Nrf2 pathway and increased expression of HO-1 in the brain, which in turn enhanced antioxidant defenses and significantly attenuated neurobehavioral deficits as well as BBB disruption in rat after stroke [45]. Similar results have been reported by Pan and fellows. They showed that SFN pretreatment significantly increased the nuclear accumulation of Nrf2 and the expression of HO-1 in ischemic retinas. And knockdown of Nrf2 with siRNA decreased cell viability and attenuated SFN-induced HO-1 up-regulation [46].

NAD(P)H: quinone oxidoreductase 1 (NQO1): In cultured astrocytes, SFN administration before or after OGD led to increased NQO1 gene and protein expression through activating Nrf2 signaling pathway; and such increased NQO1 reduced astrocyte cell death [47]. Soane, et al. [42] also found that SFN activated Nrf2 signaling pathway and induce the expression of NQO1, protecting hippocampal neurons against heme toxicity and OGD-induced death. Porritt and colleagues [48] reported that SFN induced up-regulation of NQO1 in photothrombosis-induced infarction in mice.

Other proteins: $\mathrm{Bcl}-2$ is an anti-apoptotic protein [49], and a recent report demonstrated that $\mathrm{Bcl}-2$ is a downstream gene product of Nrf2 [50]. Interestingly, Wu and colleagues reported that SFN protected cultured neurons against OGD, and protective mechanisms included that SFN up-regulated bcl-2 expression in neurons [51].

\section{Protective effects of SFN against brain diseases}

Ischemic stroke: Brain stroke is the leading cause of long-term disability and the third-leading cause of death in the entire world, and ischemic stroke is the most common type. The pathological processes of ischemic stroke are multiple, ranging from excitotoxicity, oxidative stress, inflammation, to mitochondrial dysfunction [52,53]. A number of studies have demonstrated the neuroprotective effects of SFN against ischemic brain injury through anti-oxidative mechanism.

Using a neonatal hypoxia-ischemia model, Ping and colleagues observed that SFN significantly increased Nrf2 and HO-1 expression [54]. SFN treatment decreased the number of apoptotic neurons, activated macroglia, and oxidative parameters such as the amount of 8-hydroxy-2-deoxyguanosine and malondialdehyde level, leading to a reduced brain tissue loss. The similar results have also been reported in other models. For example, SFN treatment activated the Nrf2 signaling pathway and protected cultured neurons from delayed death induced by oxygen and glucose deprivation (OGD) [42]. SFN treatment before or shortly after OGD significantly reduced astrocytes death by activating the Nrf2 pathway and antioxidant gene expression [47]. It is also demonstrated that the delayed administration of a single dose of SFN significantly decreased cerebral infarct volume in rat models of ischemia stroke $[45,55]$.

Hemorrhagic strokes: Although not as common as ischemic stroke, hemorrhagic stroke results in higher mortality rate, especially intracerebral hemorrhages (ICH) due to intracranial hypertension and brain herniation caused by hematoma and edema, ischemia and oxidative stress also contribute to brain injury after ICH [56]. SFN also exhibits neuroprotection against hemorrhagic strokes [57].

Yin and colleagues investigated the protective effects of SFN in rat ICH model [58]. They found that SFN could activate Nrf2 signaling pathway and increased HO-1 expression, and could reduce the severity of neurological dysfunction compared to control group. Similar to this study, Zhao and colleagues [59] demonstrated that SFN protected against ICH by activating Nrf2 and reducing oxidative stress and brain edema; and the deficits in Nrf2 led to weakened neuroprotective effects of SFN. It was also reported that Nrf2 activated by SFN played an essential role in the effective cleanup of the hematoma process after ICH. The authors suggested SFN could activate Nrf2 in microglia, resulting in an increase of the anti-oxidative capacity, phagocytosis and hematoma clearance after ICH $[57,59]$. Moreover, SFN treatment could ameliorate cerebral 
vasospasm and early brain injury through activation of Nrf2 signaling pathway, which in turn induced antioxidant and detoxifying enzymes in a model of subarachnoid hemorrhage $[60,61]$.

Traumatic brain injury (TBI): TBI is increasingly becoming an important cause of death, long-term disability, and post head trauma cognitive impairment around the world, with oxidative stress as an integrated pathogenesis [62]. In a rat model of TBI, SFN was able to reduce Evans Blue extravasation in the acute phase of TBI when SFN was applied before injury, and able to reduce the secondary phase of BBB permeability when administrated 6 hours after TBI [63]. The protective effects were mediated by the activation of Nrf2, indicated by increased expression of Nrf2-driven genes such as GST, HO-1, and GSH peroxidases in the cortex and cerebral micro vessels. It was also reported that intraperitoneal administration of SFN resulted in reducing neuronal death, contusion volume, and neurological dysfunction $7 \mathrm{~d}$ after TBI in rats; importantly, the neuroprotective capacity of SFN was attenuated in Nrf2 KO mice [64]. Zhang and colleagues [65] found that SFN reduced aquaporin-4 (AQP4) channel loss and increased AQP4 protein levels in the perilesion region at 24 hours and 3 days following TBI. They suggest that the neuroprotective effect of SFN may be due to a combination of mechanism, including decreased BBB permeability, enhanced cell survival, and/or increased AQP4 channel levels. SFN also exhibits protection to cognitive function after TBI. Dash and fellows [66] observed that post-injury SFN treatment enhanced learning capacity as well as improved performance of animals in a working memory task. The authors propose that the ability of SFN to protect cognitive function may be due to its ability to protect the neurons and other cell types of the neurovascular unit from the oxidative damage elicited by TBI. Taken together, SFN could protect against various pathophysiological consequences of TBI, in which activating Nrf2 pathway plays a key role [67].

Alzheimer's disease (AD): $\mathrm{AD}$ is the commonest cause of dementia without a cure. It is characterized by memory loss and cognitive dysfunction, and its pathological hallmarks are the cerebral deposition of amyloid-beta $(\mathrm{A} \beta)$ peptides in senile plaques and the neurofibrillary tangles of hyper-phosphorylated tau aggregates $[4,68,69]$. $A \beta$ is believed to bean important reason for neuronal cell death in $\mathrm{AD}$, and $\mathrm{A} \beta 40$ and $\mathrm{A} \beta 42$ peptides can exist in multiple aggregation forms, including soluble oligomers or protofibrils, to insoluble fibrils, responsible for various pathological effects [70]. A $\beta$ has been reported to induce the production of hydrogen peroxides in cultured cells [71]; on the other hand, oxidative production increases $\mathrm{A} \beta$ production [72]. As discussed below, SFN demonstrates its protective effects against $\mathrm{AD}$, due to its ability to activate $\mathrm{Nrf} 2$ and consequent antioxidant.

SFN was reported to be able to ameliorate cognitive deficit in various models of $\mathrm{AD}[69,73]$. Lee and colleagues [74] demonstrated that SFN protected $A \beta$-induced oxidative death of cultured neuronal cells. Park, et al. confirmed the ability of SFN to protect the neuronal cells from $A \beta 42$-mediated cytoxicity [75]. In an $A \beta$-induced mouse model of $\mathrm{AD}$, Kim, et al. reported that $\mathrm{SFN}$ could ameliorate cognitive impairment and protect the brain from amyloidogenic damages [69]. $\mathrm{A} \beta$ peptides are degraded, at least in part, through autophagy. A recent study showed that Nrf2 promotes autophagy by upregulation of p62 protein, an adaptor for selective autophagy [76]. By administration of
SFN, autophagy genes was up regulated in cultured cells; when Nrf2 was knocked out, SFN exhibited a reduced induction of these genes [77].

However, it is not clear whether SFN can protect the brain against vascular cognitive impairment and dementia, the second commonest type of dementia.

Parkinson's Disease (PD): Oxidative stress is an important factor in the pathogenesis of PD [78]. A number of studies showed that SFN was able to significantly protect dopaminergic cells from the cytotoxicity of 6-OHDA [79,80]. Vouzour, et al. [43] have found that SFN could protect primary cortical neurons against 5-S-cysteinyldopamine-induced injury, which is formed by DA quinone reacting with cellular thiols. It was reported that SFN inhibited 6-OHDAinduced cytotoxicity in dopaminergic-like neuroblastoma SH-SY5Y cells through increasing Nrf2 nuclear translocation, and increasing the levels of HO-1 expression and GSH contents [81,82]. Other studies confirmed that Nrf2 activation by SF might play an important role in DA neuron protection against 6-OHDA-induced toxicity in rat organo typical nigrostriatal coculture [83]. In vivo, SFN could also reduce neurotoxicity induced by rotenone, where Nrf2 played a key role [84]. In summary, SFN protects against $\mathrm{PD}$, mainly through the Nrf2 signaling pathway.

Autism Spectrum Disorders (ASD): ASD refers a group of neurodevelopmental disorders, characterized by deficits in communicating with others and repeated behaviors staring in early childhood. The pathogenesis of ASD is not clear now; however, several causes have been suggested for ASD, including increased oxidative stress and decreased cellular defense [85,86]. A recent clinical study suggested SFN treatment improved the social interaction, abnormal behavior, and verbal communication of the patients [87]. In addition, a phase 2 clinical trial (NCT02561481) is ongoing to detect the effects on SFN on ASD.

\section{Conclusion}

Along with other beneficial effects of SFN, such as antiinflammation [88]. Multiple in vitro and in vivo studies have demonstrated the ability of SFN to prevent various brain diseases, including stroke, TBI, AD, PD, and ASD. SFN exerts neuroprotective effects, at least in a part, through activating Nrf2 signaling pathway and its downstream antioxidative as well as antitoxic enzymes. Existing in various vegetables especially in broccoli, SFN can be easily consumed as a phytochemical inducer of Nrf2. Because of its BBB permeability, SFN can reach effective concentration in the CNS. Considering its other beneficial effects, such as anti-inflammation [88], SFN is a promising dietary and medical agent for neuroprotection.

\section{Acknowledgement}

This work was supported by grants from the National Institutes of Health (NS092810 to F.Z.), and the Natural Science Foundation of China (81271276 to F.Z.). We thank Miaoyuan Jessica Zhang for editorial support and Patricia Strickler for administrative support.

\section{References}

1. Mandel S, Grünblatt E, Riederer P, Gerlach M, Levites Y, Youdim MB. Neuroprotective strategies in Parkinson's disease: an update on progress. CNS Drugs. 2003; 17: 729-762. 
2. Harris RA, Amor S. Sweet and sour--oxidative and carbonyl stress in neurological disorders. CNS Neurol Disord Drug Targets. 2011; 10: 82-107.

3. Tarozzi A, Angeloni C, Malaguti M, Morroni F, Hrelia S, Hrelia P. Sulforaphane as a potential protective phytochemical against neurodegenerative diseases. Oxid Med Cell Longev. 2013; 2013: 415078.

4. van Muiswinkel FL, Kuiperij HB. The Nrf2-ARE Signalling pathway: promising drug target to combat oxidative stress in neurodegenerative disorders. Curr Drug Targets CNS Neurol Disord. 2005; 4:267-81.

5. Sun Y, Yang T, Leak RK, Chen JH, Zhang F. Preventive and protective roles of dietary Nrf2 activators against central nervous system diseases. CNS Neurol Disord Drug Targets. 2017.

6. Ghawi SK, Methven L, Niranjan K. The potential to intensify sulforaphane formation in cooked broccoli (Brassica oleracea var. italica) using mustard seeds (Sinapis alba). Food Chem. 2013; 138: 1734-1741.

7. Hwang ES, Jeffery EH. Induction of quinone reductase by sulforaphane and sulforaphane $\mathrm{N}$-acetylcysteine conjugate in murine hepatoma cells. J Med Food. 2005; 8: 198-203.

8. McNaughton SA, Marks GC. Development of a food composition database for the estimation of dietary intakes of glucosinolates, the biologically active constituents of cruciferous vegetables. Br J Nutr. 2003; 90: 687-697.

9. Sones K, Heaney RK, Fenwick GR. An Estimate of the Mean Daily Intake of Glucosinolates from Cruciferous Vegetables in the Uk. J Sci Food Agr. 1984 35: 712-720.

10. Totusek J, Triska J, Lefnerova D, Strohalm J, Vrchotova N, Zendulka O, et al. Contents of Sulforaphane and Total Isothiocyanates, Antimutagenic Activity, and Inhibition of Clastogenicity in Pulp Juices from Cruciferous Plants. Czech J Food Sci. 2011; 29: 548-556.

11. Xie S, He J, He J. Determining sulforaphane contents in edible parts of nine cruciferous vegetables. J Anhui Agricultural Univ. 2013; 40: 460-463.

12. Fahey JW, Zalcmann AT, Talalay P. The chemical diversity and distribution of glucosinolates and isothiocyanates among plants. Phytochemistry. 2001; 56: 5-51

13. Keum YS. Regulation of the Keap1/Nrf2 system by chemopreventive sulforaphane: implications of posttranslational modifications. Ann N Y Acad Sci. 2011; 1229: 184-189.

14. Egner PA, Chen JG, Wang JB, Wu Y, Sun Y, Lu JH, et al. Bioavailability of Sulforaphane from two broccoli sprout beverages: results of a short-term, cross-over clinical trial in Qidong, China. Cancer Prev Res (Phila). 2011; 4 384-395

15. Kensler TW, Wakabayashi N, Biswal S. Cell survival responses to environmental stresses via the Keap1-Nrf2-ARE pathway. Annu Rev Pharmacol Toxicol. 2007; 47: 89-116.

16. Hu R, Hebbar V, Kim BR, Chen C, Winnik B, Buckley B, et al. In vivo pharmacokinetics and regulation of gene expression profiles by isothiocyanate sulforaphane in the rat. J Pharmacol Exp Ther. 2004; 310: 263-271.

17. Ye L, Dinkova-Kostova AT, Wade KL, Zhang Y, Shapiro TA, Talalay P. Quantitative determination of dithiocarbamates in human plasma, serum, erythrocytes and urine: pharmacokinetics of broccoli sprout isothiocyanates in humans. Clin Chim Acta. 2002; 316: 43-53.

18. Jazwa A, Rojo AI, Innamorato NG, Hesse M, Fernandez-Ruiz J, Cuadrado A Pharmacological targeting of the transcription factor Nrf2 at the basal ganglia provides disease modifying therapy for experimental parkinsonism. Antioxid Redox Signal. 2011; 14: 2347-2360.

19. Clarke JD, Hsu A, Williams DE, Dashwood RH, Stevens JF, Yamamoto M, et al. Metabolism and tissue distribution of sulforaphane in Nrf2 knockout and wild-type mice. Pharm Res. 2011; 28: 3171-3179.

20. Zhang M, An C, Gao Y, Leak RK, Chen J, Zhang F. Emerging roles of Nrf2 and phase II antioxidant enzymes in neuroprotection. Prog Neurobiol. 2013; 100: $30-47$.

21. Eggler AL, Gay KA, Mesecar AD. Molecular mechanisms of natural products in chemoprevention: induction of cytoprotective enzymes by Nrf2. Mol Nutr
Food Res. 2008; 52 Suppl 1: S84-S94.

22. Stefanson AL, Bakovic M. Dietary regulation of Keap1/Nrf2/ARE pathway: focus on plant-derived compounds and trace minerals. Nutrients. 2014; 6 : 3777-3801.

23. McMahon M, Thomas N, Itoh K, Yamamoto M, Hayes JD. Dimerization of substrate adaptors can facilitate cullin-mediated ubiquitylation of proteins by a "tethering" mechanism: a two-site interaction model for the Nrf2-Keap1 complex. J Biol Chem. 2006; 281: 24756-24768.

24. Itoh K, Chiba T, Takahashi S, Ishii T, Igarashi K, Katoh Y, et al. An Nrf2/small Maf heterodimer mediates the induction of phase II detoxifying enzyme genes through antioxidant response elements. Biochem Biophys Res Commun. 1997; 236: 313-322.

25. Rushmore TH, Morton MR, Pickett $\mathrm{CB}$. The antioxidant responsive element Activation by oxidative stress and identification of the DNA consensus sequence required for functional activity. J Biol Chem. 1991; 266: 1163211639

26. Nguyen T, Sherratt PJ, Huang HC, Yang CS, Pickett CB. Increased protein stability as a mechanism that enhances Nrf2-mediated transcriptional activation of the antioxidant response element. Degradation of Nrf2 by the 26 S proteasome. J Biol Chem. 2003; 278: 4536-4541.

27. Motohashi H, O'Connor T, Katsuoka F, Engel JD, Yamamoto M. Integration and diversity of the regulatory network composed of Maf and CNC families of transcription factors. Gene. 2002; 294: 1-12.

28. Rada P, Rojo Al, Evrard-Todeschi N, Innamorato NG, Cotte A, Jaworski T, et al. Structural and Functional Characterization of Nrf2 Degradation by the Glycogen Synthase Kinase 3/ß-TrCP Axis. Mol Cell Biol. 2012; 32: 34863499.

29. Rada P, Rojo Al, Chowdhry S, McMahon M, Hayes JD, Cuadrado A. SCF/ßTrCP Promotes Glycogen Synthase Kinase 3-Dependent Degradation of the Nrf2 Transcription Factor in a Keap1-Independent Manner. Mol Cell Biol. 2011; 31: 1121-1133.

30. Su ZY, Zhang C, Lee JH, Shu L, Wu TY, Khor TO, et al. Requirement and epigenetics reprogramming of Nrf2 in suppression of tumor promoter TPAinduced mouse skin cell transformation by sulforaphane. Cancer Prev Res (Phila). 2014; 7: 319-329.

31. Bai Y, Cui W, Xin Y, Miao X, Barati MT, Zhang C, et al. Prevention by sulforaphane of diabetic cardiomyopathy is associated with up-regulation of Nrf2 expression and transcription activation. J Mol Cell Cardiol. 2013;57: 8295.

32. McMahon M, Lamont DJ, Beattie KA, Hayes JD. Keap1 perceives stress via three sensors for the endogenous signaling molecules nitric oxide, zinc, and alkenals. Proc Natl Acad Sci U S A. 2010; 107: 18838-18843.

33. Hu R, Xu C, Shen G, Jain MR, Khor TO, Gopalkrishnan A, et al. Gene expression profiles induced by cancer chemopreventive isothiocyanate sulforaphane in the liver of C57BL/6J mice and C57BL/6J/Nrf2 (-/-) mice. Cancer Lett. 2006; 243: 170-192.

34. Hu C, Eggler AL, Mesecar AD, van Breemen RB. Modification of keap1 cysteine residues by sulforaphane. Chem Res Toxicol. 2011; 24: 515-521.

35. Hong F, Freeman ML, Liebler DC. Identification of sensor cysteines in human Keap1 modified by the cancer chemopreventive agent sulforaphane. Chemical research in toxicology. 2005; 18: 1917-1926.

36. Shang GG, Tang XJ, Gao P, Guo FL, Liu HP, Zhao ZH, et al. Sulforaphane attenuation of experimental diabetic nephropathy involves GSK-3 beta/Fyn/ Nrf2 signaling pathway. Journal of Nutritional Biochemistry. 2015; 26: 596606.

37. McMahon M, Itoh K, Yamamoto M, Hayes JD. Keap1-dependent proteasomal degradation of transcription factor Nrf2 contributes to the negative regulation of antioxidant response element-driven gene expression. J Biol Chem. 2003; 278: 21592-21600.

38. Bergstrom P, Andersson HC, Gao Y, Karlsson JO, Nodin C, Anderson MF, et al. Repeated transient sulforaphane stimulation in astrocytes leads to prolonged Nrf2-mediated gene expression and protection from superoxide- 
induced damage. Neuropharmacology. 2011; 60: 343-353.

39. Angeloni C, Leoncini E, Malaguti M, Angelini S, Hrelia P, Hrelia S. Modulation of phase II enzymes by sulforaphane: implications for its cardioprotective potential. J Agric Food Chem. 2009; 57: 5615-5622.

40. Griffith OW. Biologic and pharmacologic regulation of mammalian glutathione synthesis. Free Radic Biol Med. 1999; 27: 922-935

41. Franklin CC, Backos DS, Mohar I, White CC, Forman HJ, Kavanagh TJ. Structure, function, and post-translational regulation of the catalytic and modifier subunits of glutamate cysteine ligase. Mol Aspects Med. 2009; 30: 86-98.

42. Soane L, Li Dai W, Fiskum G, Bambrick LL. Sulforaphane protects immature hippocampal neurons against death caused by exposure to hemin or to oxygen and glucose deprivation. J Neurosci Res. 2010; 88: 1355-1363.

43. Vauzour D, Buonfiglio M, Corona G, Chirafisi J, Vafeiadou K, Angeloni C, e al. Sulforaphane protects cortical neurons against 5-S-cysteinyl-dopamineinduced toxicity through the activation of ERK1/2, Nrf-2 and the upregulation of detoxification enzymes. Mol Nutr Food Res. 2010; 54: 532-542.

44. Ferrándiz ML, Devesa I. Inducers of heme oxygenase-1. Curr Pharm Des. 2008; 14: 473-486.

45. Alfieri A, Srivastava S, Siow RC, Cash D, Modo M, Duchen MR, et al Sulforaphane preconditioning of the $\mathrm{Nr} 2 / \mathrm{HO}-1$ defense pathway protects the cerebral vasculature against blood-brain barrier disruption and neurological deficits in stroke. Free Radic Biol Med. 2013; 65:1012-1022.

46. Lee YJ, Jeong HY, Kim YB, Lee YJ, Won SY, Shim JH, et al. Reactive oxygen species and PI3K/Akt signaling play key roles in the induction of Nrf2-driven heme oxygenase-1 expression in sulforaphane-treated human mesothelioma MSTO-211H cells. Food Chem Toxicol. 2012; 50: 116-123.

47. Danilov CA, Chandrasekaran K, Racz J, Soane L, Zielke C, Fiskum G. Sulforaphane protects astrocytes against oxidative stress and delayed death caused by oxygen and glucose deprivation. Glia. 2009; 57: 645-656.

48. Porritt MJ, Andersson HC, Hou L, Nilsson $\AA$, Pekna M, Pekny M, et al. Photothrombosis-induced infarction of the mouse cerebral cortex is not affected by the Nrf2-activator sulforaphane. PLoS One. 2012; 7: e41090.

49. Zhang F, Yin W, Chen J. Apoptosis in cerebral ischemia: executional and regulatory signaling mechanisms. Neurol Res. 2004; 26: 835-845.

50. Niture SK, Jaiswal AK. Nrf2 protein up-regulates antiapoptotic protein Bcl-2 and prevents cellular apoptosis. J Biol Chem. 2012; 287: 9873-9886.

51. Wu X, Zhao J, Yu S, Chen Y, Wu J, Zhao Y. Sulforaphane protects primary cultures of cortical neurons against injury induced by oxygen-glucose deprivation/reoxygenation via antiapoptosis. Neurosci Bull. 2012; 28: 509516

52. Rodrigo R, Fernandez-Gajardo R, Gutierrez R, Matamala JM, Carrasco R Miranda-Merchak A, et al. Oxidative stress and pathophysiology of ischemic stroke: novel therapeutic opportunities. CNS Neurol Disord Drug Targets. 2013; 12: 698-714.

53. Ahmad M, Dar NJ, Bhat ZS, Hussain A, Shah A, Liu H, et al. Inflammation in ischemic stroke: mechanisms, consequences and possible drug targets. CNS Neurol Disord Drug Targets. 2014; 13: 1378-1396.

54. Ping Z, Liu W, Kang Z, Cai J, Wang Q, Cheng N, et al. Sulforaphane protects brains against hypoxic-ischemic injury through induction of Nrf2-dependent phase 2 enzyme. Brain Res. 2010; 1343: 178-185.

55. Zhao J, Kobori N, Aronowski J, Dash PK. Sulforaphane reduces infarct volume following focal cerebral ischemia in rodents. Neurosci Lett. 2006; 393: 108-112.

56. Keep RF, Hua Y, Xi G. Intracerebral haemorrhage: mechanisms of injury and therapeutic targets. Lancet Neurol. 2012; 11: 720-731.

57. Zhao X, Sun G, Ting SM, Song S, Zhang J, Edwards NJ, et al. Cleaning up after $\mathrm{ICH}$ : the role of $\mathrm{Nrf} 2$ in modulating microglia function and hematoma clearance. J Neurochem. 2015; 133: 144-152.

58. Yin XP, Chen ZY, Zhou J, Wu D, Bao B. Mechanisms underlying the perifocal neuroprotective effect of the Nrf2-ARE signaling pathway after intracranial hemorrhage. Drug Des Devel Ther. 2015; 9: 5973-5986.

59. Zhao X, Sun G, Zhang J, Strong R, Dash PK, Kan YW, et al. Transcription factor Nrf2 protects the brain from damage produced by intracerebral hemorrhage. Stroke. 2007; 38: 3280-3286.

60. Chen G, Fang Q, Zhang J, Zhou D, Wang Z. Role of the Nrf2-ARE pathway in early brain injury after experimental subarachnoid hemorrhage. J Neurosci Res. 2011; 89: 515-523.

61. Zhao X, Wen L, Dong M, Lu X. Sulforaphane activates the cerebral vascular Nrf2-ARE pathway and suppresses inflammation to attenuate cerebral vasospasm in rat with subarachnoid hemorrhage. Brain Res. 2016; 1653: 1-7.

62. Masel BE, DeWitt DS. Traumatic brain injury: a disease process, not an event. J Neurotrauma. 2010; 27: 1529-1540.

63. Zhao J, Moore AN, Redell JB, Dash PK. Enhancing expression of Nrf2-driven genes protects the blood brain barrier after brain injury. J Neurosci. 2007; 27:10240-10248.

64. Hong Y, Yan W, Chen S, Sun CR, Zhang JM. The role of Nrf2 signaling in the regulation of antioxidants and detoxifying enzymes after traumatic brain injury in rats and mice. Acta Pharmacol Sin. 2010; 31: 1421-1430.

65. Zhao J, Moore AN, Clifton GL, Dash PK. Sulforaphane enhances aquaporin-4 expression and decreases cerebral edema following traumatic brain injury. J Neurosci Res. 2005; 82: 499-506

66. Dash PK, Zhao J, Orsi SA, Zhang M, Moore AN. Sulforaphane improves cognitive function administered following traumatic brain injury. Neurosci Lett. 2009; 460: 103-107.

67. Fernandez-Gajardo R, Matamala JM, Carrasco R, Gutierrez R, Melo R, Rodrigo R. Novel therapeutic strategies for traumatic brain injury: acute antioxidant reinforcement. CNS Drugs. 2014; 28: 229-248.

68. Klein WL, Krafft GA, Finch CE. Targeting small Abeta oligomers: the solution to an Alzheimer's disease conundrum? Trends Neurosci. 2001; 24: 219-224.

69. Kim HV, Kim HY, Ehrlich HY, Choi SY, Kim DJ, Kim Y. Amelioration of Alzheimer's disease by neuroprotective effect of sulforaphane in animal model. Amyloid. 2013; 20: 7-12.

70. Tarozzi A, Morroni F, Merlicco A, Bolondi C, Teti G, Falconi M, et al. Neuroprotective effects of cyanidin 3-O-glucopyranoside on amyloid beta (25-35) oligomer-induced toxicity. Neurosci Lett. 2010; 473: 72-76.

71. Andersen JK. Oxidative stress in neurodegeneration: cause or consequence? Nat Med. 2004; S18-S25.

72. Leuner K, Schutt T, Kurz C, Eckert SH, Schiller C, Occhipinti A, et al. Mitochondrion-derived reactive oxygen species lead to enhanced amyloid beta formation. Antioxid Redox Signal. 2012; 16: 1421-1433.

73. Zhang R, Miao QW, Zhu CX, Zhao Y, Liu L, Yang J, et al. Sulforaphane ameliorates neurobehavioral deficits and protects the brain from amyloid beta deposits and peroxidation in mice with Alzheimer-like lesions. Am J Alzheimers Dis Other Demen. 2015; 30: 183-191.

74. Lee C, Park GH, Lee SR, Jang JH. Attenuation of $\beta$-amyloid-induced oxidative cell death by sulforaphane via activation of NF-E2-related factor 2. Oxid Med Cell Longev. 2013; 2013: 313510.

75. Park HM, Kim JA, Kwak MK. Protection against amyloid beta cytotoxicity by sulforaphane: role of the proteasome. Arch Pharm Res. 2009; 32: 109-115

76. Fujita KI, Maeda D, Xiao Q, Srinivasula SM. Nrf2-mediated induction of p62 controls Toll-like receptor-4-driven aggresome-like induced structure formation and autophagic degradation. Proc Natl Acad Sci U S A. 2011; 108 : $1427-1432$.

77. Pajares M, Jiménez-Moreno N, García-Yagüe ÁJ. Transcription factor NFE2L2/NRF2 is a regulator of macroautophagy genes. Autophagy. 2016; 12: $1902-1916$.

78. Kumar H, Koppula S, Kim IS, More SV, Kim BW, Choi DK. Nuclear factor erythroid 2-related factor 2 signaling in Parkinson disease: a promising multi therapeutic target against oxidative stress, neuroinflammation and cell death. CNS Neurol Disord Drug Targets. 2012; 11: 1015-1029. 
79. Han JM, Lee YJ, Lee SY, Kim EM, Moon Y, Kim HW, et al. Protective effect of sulforaphane against dopaminergic cell death. J Pharmacol Exp Ther. 2007; 321: $249-256$.

80. Yoon NS, Cho Y, Lee SY, Choi HJ, Hwang O. Inactivation of Aconitase by Tetrahydrobiopterin in DArgic Cells: Relevance to PD. Exp Neurobiol. 2010; 19: 23-29.

81. Tarozzi A, Morroni F, Merlicco A, Hrelia S, Angeloni C, Cantelli-Forti G, et al. Sulforaphane as an inducer of glutathione prevents oxidative stress-induced cell death in a dopaminergic-like neuroblastoma cell line. J Neurochem. 2009; 111: $1161-1171$

82. Deng C, Tao R, Yu SZ, Jin H. Sulforaphane protects against 6-hydroxydopamine-induced cytotoxicity by increasing expression of heme oxygenase-1 in a PI3K/Akt-dependent manner. Mol Med Rep. 2012; 5: 847851

83. Siebert A, Desai V, Chandrasekaran K, Fiskum G, Jafri MS. Nrf2 activators provide neuroprotection against 6-hydroxydopamine toxicity in rat organotypic nigrostriatal cocultures. J Neurosci Res. 2009; 87: 1659-1669.

84. Zhou Q, Chen B, Wang X, Wu L, Yang Y, Cheng X, et al. Sulforaphane protects against rotenone-induced neurotoxicity in vivo: Involvement of the mTOR, Nrf2, and autophagy pathways. Sci Rep. 2016; 6: 32206.

85. Liu H, Talalay P, Fahey JW. Biomarker-Guided Strategy for Treatment of Autism Spectrum Disorder (ASD). CNS Neurol Disord Drug Targets. 2016 15: 602-613.

86. Calabrese V, Giordano J, Ruggieri M, Berritta D, Trovato A, Ontario ML, et al. Hormesis, cellular stress response, and redox homeostasis in autism spectrum disorders. J Neurosci Res. 2016; 94: 1488-1498.

87. Singh K, Connors SL, Macklin EA, Smith KD, Fahey JW, Talalay P, et al. Sulforaphane treatment of autism spectrum disorder (ASD). Proceedings of the National Academy of Sciences of the United States of America. 2014; 111: 15550-15555.

88. Holloway PM, Gillespie S, Becker F, Vital SA, Nguyen V, Alexander JS, et al. Sulforaphane induces neurovascular protection against a systemic inflammatory challenge via both Nrf2-dependent and independent pathways. Vasc Pharmacol. 2016; 85:29-38.
Austin J Cerebrovasc Dis \& Stroke - Volume 4 Issue 1 - 2017 ISSN : 2381-9103 | www.austinpublishinggroup.com

Zhang et al. (C) All rights are reserved
Citation: Sun Y, Yang T, Mao L and Zhang F. Sulforaphane Protects against Brain Diseases: Roles of Cytoprotective Enzymes. Austin J Cerebrovasc Dis \& Stroke. 2017; 4(1): 1054. 